\title{
REALIZABILITY OF BRANCHED COVERINGS OF SURFACES
}

\author{
BY
}

ALLAN L. EDMONDS, RAVI S. KULKARNI AND ROBERT E. STONG

\begin{abstract}
A branched covering $M \rightarrow N$ of degree $d$ between closed surfaces determines a collection $D$ of partitions of $d$-its "branch data"-corresponding to the set of branch points. The collection of partitions must satisfy certain obvious conditions implied by the Riemann-Hurwitz formula. This paper investigates the extent to which any such finite collection oD of partitions of $d$ can be realized as the branch data of a suitable branched covering. If $N$ is not the 2-sphere, such data can always be realized. If ol contains sufficiently many elements compared to $d$, then it can be realized. And whenever $d$ is nonprime, examples are constructed of nonrealizable data.
\end{abstract}

1. Introduction. Let $M$ and $N$ denote closed, connected surfaces. A smooth map $\phi$ : $M \rightarrow N$ is a degree $d$ branched covering if for each $x \in N$ there is a partition $A(x)=\left[a_{1}, \ldots, a_{r}\right]$ of $d$ such that, over a neighborhood of $x$ in $N, \phi$ is equivalent to the map $f:\{1, \ldots, r\} \times \mathbf{C} \rightarrow \mathbf{C}$ where $f(i, z)=z^{a_{i}}$ and $x$ corresponds to 0 in $\mathbf{C}$. The set of points $x \in N$ for which $A(x)$ is not the trivial partition $[1, \ldots, 1]$ of $d$ constitutes the branch set $B_{\phi}$ of $\phi$. The collection $\mathscr{D}=\left\{A(x): x \in B_{\phi}\right\}$ (with repetitions allowed) is called the branch data of $\phi$.

This paper is addressed to the following question.

Realizability Problem. Given a closed, connected surface $N$ and a collection O $=\left\{A_{1}, \ldots, A_{k}\right\}$ of partitions of a positive integer $d$, is there a branched covering $\phi: M \rightarrow N$ with $\mathscr{D}$ as its branch data?

The history of the problem goes back to Hurwitz [8], who essentially showed how to reduce the general question to a problem about realizing partitions by suitable permutations in the symmetric group $\Sigma_{d}$. To describe this in the case $N$ is the 2-sphere $S^{2}$, recall that each element $\alpha \in \Sigma_{d}$ may be written uniquely as a product $\alpha=\gamma_{1} \cdots \gamma_{r}$ of disjoint cycles (including cycles of length 1 if necessary). Then $\alpha$ determines the partition $A=\|\alpha\|=\left[\left|\gamma_{1}\right|, \ldots,\left|\gamma_{r}\right|\right]$ given by the lengths $\left|\gamma_{i}\right|$ of these cycles and one writes $\alpha \in A$. Two elements of $\Sigma_{d}$ are conjugate if and only if they have the same cycle lengths; thus the set of partitions of $d, \pi(d)$, is identified with the set of conjugacy classes in $\Sigma_{d}$. Now a collection $\mathscr{D}=\left\{A_{1}, \ldots, A_{k}\right\}$ of partitions of $d$ is the branch data for a connected branched covering of $S^{2}$ if and only if there exist $\alpha_{1}, \ldots, \alpha_{k} \in \Sigma_{d}$ such that $\alpha_{i} \in A_{i}, 1 \leqslant i \leqslant k$; and $\alpha_{1} \cdots \alpha_{k}=1$ and the subgroup $\left\langle\alpha_{1}, \ldots, \alpha_{k}\right\rangle$ of $\Sigma_{d}$ generated by $\left\{\alpha_{1}, \ldots, \alpha_{k}\right\}$ acts transitively on $\{1, \ldots, d\}$. For more details see $\S 2$.

It turns out to be a very delicate problem, in general, to decide whether or not there exist such elements in $\Sigma_{d}$ for given $\mathscr{D}$. There are some easy and well-known

Received by the editors September 20, 1982 and, in revised form, May 10, 1983.

1980 Mathematics Subject Classification. Primary 57M12.

(C) 1984 American Mathematical Society $0002-9947 / 84 \$ 1.00+\$ .25$ per page 
necessary conditions, which are here referred to as the Hurwitz conditions, and which are essentially consequences of the Riemann-Hurwitz formula:

If $\phi: M \rightarrow N$ is a degree $d$ branched covering of closed, connected surfaces with branch data $\mathscr{D}=\left\{A_{1}, \ldots, A_{k}\right\}$, then the total branching $v(\phi)=v(\mathscr{D})=\sum_{i=1}^{k} v\left(A_{i}\right)$ is even; where if $A=\left[a_{1}, \ldots, a_{r}\right] \in \pi(d)$, then $v(A)=d-r=\sum_{i=1}^{r}\left(a_{i}-1\right)$. If $N$ is orientable, then so is $M$; if $d$ is odd and $N$ is nonorientable, then $M$ is also nonorientable. And finally, $\chi(M)=d \chi(N)-v(\phi)$, where $\chi(M) \leqslant 2$ if $M$ is orientable and $\chi(M) \leqslant 1$ if $M$ is nonorientable. If $M$ is orientable while $N$ is not, then $\phi$ lifts to a branched covering $\tilde{\phi}: M \rightarrow \tilde{N}$, where $\tilde{N}$ is the orientable double cover of $N$; it then follows that each $A_{i}=\left[B_{i}, C_{i}\right]$ where $B_{i}$ and $C_{i}$ are partitions of $d / 2$ and $\tilde{\text { OD }}=\left\{B_{1}, \ldots, B_{k}, C_{1}, \ldots, C_{k}\right\}$ is the branch data for $\tilde{\phi}$.

When $\chi(N) \leqslant 0$ these conditions are actually also sufficient. The proof is rather straightforward and is given in $\S 3$. This result is for the most part previously known. The case when $N$ is orientable is given in Husemoller's thesis [7]. The case when $N$ is nonorientable was proved by Ezell [5]. Ezell, however, did not distinguish the cases when $M$ can be chosen to be orientable.

Further results stated below require a more detailed study of certain products in $\Sigma_{d}$, which is given in $\S 4$.

THEOREM. If $N$ is the projective plane and $\mathscr{D}$ is a collection of partitions of $d$, then there is a degree $d$ branched covering $\phi: M \rightarrow N$ with $M$ closed, connected, and nonorientable, and with branch data $\mathcal{D}$ if and only if $v(\mathcal{D})$ is even and $v(\mathcal{D}) \geqslant d-1$.

The proof is completed in $\$ 5$. These results reduce the central problem to the consideration of branched coverings of the 2-sphere $S^{2}$. (The orientable branched coverings of $\mathbf{R} P^{2}$ are actually branched coverings of $S^{2}$, with some extra difficulty related to choosing refinements $A=[B, C]$ for partitions $A$.) The case $N=S^{2}$ is very difficult: the necessary Hurwitz conditions are not sufficient, in general, and a complete solution of the realization problem remains elusive.

Fix an integer $d \geqslant 2$ and let $\Delta(d)$ denote the set of collections $\mathscr{D}=\left\{A_{1}, \ldots, A_{k}\right\}$ of putative branch data satisfying the Hurwitz conditions that $v(\mathscr{D})=\Sigma v\left(A_{i}\right)$ is even and $v(\mathscr{D}) \geqslant 2(d-1)$.

THEOREM. If $d$ is not prime, then there exists $\mathscr{D} \in \Delta(d)$ which is not the branch data for a connected branched covering of $S^{2}$.

THEOREM. If $d \neq 4$, then there are at most finitely many elements of $\Delta(d)$ which are not realizable as the branch data for a connected branched covering of $S^{2}$. In particular, if $v(\mathscr{D}) \geqslant n(d)=3(d-1)$ then $\mathscr{D}$ is realizable.

These results are also proved in $\$ 5$. The elements of $\Delta(4)$ are realizable with the exception of the sequence of data of the form $\{[3,1],[2,2], \ldots,[2,2]\}$. If $d=2,3,5$ or 7 every element of $\Delta(d)$ is realizable. Similar assertions also hold for orientable branched coverings of $\mathbf{R} P^{2}$.

These theorems suggest several questions.

(1) To what extent can the bound $n(d)=3(d-1)$ above be improved? In particular, if $d$ is prime, is every element of $\Delta(d)$ realizable by a connected branched covering of $S^{2}$ ? 
(2) Can one find further reasonably simple conditions which guarantee the realizability of given branch data?

(3) Can one find reasonably stated additional conditions which must be satisfied by the branch data of branched coverings of $S^{2}$ ?

As for question (1), the results of $\S 5$ reduce the problem when $d$ is prime to the consideration of the 3 branch point case. But to date no conclusive results have been obtained.

As an example of an answer to question (2) we have obtained a complete determination of the realizability of branch data of the form $\mathscr{D}=\left\{\left[a_{1}, \ldots, a_{r}\right]\right.$, $\left.\left[b_{1}, \ldots, b_{s}\right],[m, 1, \ldots, 1]\right\}$. $\mathcal{D}$ is realizable if and only if $v(\mathscr{D})$ is even, $v(\mathscr{D}) \geqslant 2(d-1)$, and, if $v(\mathcal{D})=2(d-1)$, then $m \leqslant d / \operatorname{GCD}\left(a_{1}, \ldots, a_{r}, b_{1}, \ldots, b_{s}\right)$. An equivalent statement has been discovered independently and published by Boccara [2]. No further details will be given here.

As an example of an additional condition, the following result is derived in $§ 6$.

THEOREM. Let $\mathscr{D}=\left\{A_{1}, \ldots, A_{r}\right\}$ be a collection of partitions of $d$ with $v(\mathscr{D})=2 d-$ 2 , and $A_{i}=\left[a_{i 1}, \ldots, a_{i r_{1}}\right], 1 \leqslant i \leqslant k$. If $\mathscr{D}$ is realizable as the branch data of $a$ connected branched covering of $S^{2}$, then

$$
\sum_{i=1}^{k} \sum_{m=1}^{r_{t}}\left[d-\sum_{n=1}^{r_{i}} \operatorname{gcd}\left(a_{i m}, a_{i n}\right)\right] \geqslant 2\left[d-\min _{i, m} \sum_{n=1}^{r_{i}} \operatorname{gcd}\left(a_{i m}, a_{i n}\right)\right] .
$$

For $d$ a prime this inequality is always satisfied, but for certain rather limited families of data it does provide nontrivial restrictions. See $\S 6$.

In conclusion, it should be noted that there is a very close connection between the problem addressed in this paper and the problem of determining the subgroups $\Phi$ of finite index $d$ in a given Fuchsian group $\Gamma$. If $\Gamma$ acts on the complex upper halfplane $H$, then passing to quotient spaces yields a branched covering $\Phi \backslash H \rightarrow \Gamma \backslash H$. If one fixes $\Gamma$, then the subgroups $\Phi$ of index $d$ correspond to branch data satisfying certain extra conditions. See Singerman [12] and also Edmonds, Ewing and Kulkarni [3, 4]. The present authors plan to address in a sequel to this paper the realizability problem from this point of view, emphasizing the fundamental case of three branch points. ${ }^{1}$

ACKNOWLEDGEMENTS. All three authors received financial support from the National Science Foundation. The second author acknowledges the support of the Guggenheim Foundation and the hospitality of the University of Colorado where part of this work was done. The third author is indebted to Leonard Scott for many conversations about this material.

2. The Hurwitz conditions. Let $\phi: M \rightarrow N$ be a degree $d$ branched covering of closed, connected surfaces. Clearly the branch points in $N$ are isolated, and hence $B_{\phi}$ is a finite set. The branched covering $\phi$ is completely determined by the restriction

\footnotetext{
${ }^{1}$ An exposition of this work and other related material appears in Surface-Symmetries, Holomorphic Maps and Tessellations by Ravi S. Kulkarni, Differential Geometry Proceedings, Special Year, Maryland 1981-82 (ed. Brooks, Gray, Reinhart), Progress in Math., vol. 32, Birkhäuser, 1983, pp. 162-176.
} 
$\phi_{0}: M-\phi^{-1} B_{\phi} \rightarrow N-B_{\phi}$, an ordinary, connected $d$-fold covering space. For given a connected $d$-fold covering space $\psi: P \rightarrow N-F$ where $F \subset N$ is a finite set, covering space theory applied to small circles about the elements of $F$ shows that $\psi$ extends to a branched covering $M \rightarrow N$ where $M$ is just the end compactification of $P$, and is a closed, connected surface.

If $\psi: P \rightarrow N-F$ is a $d$-fold covering space, one may fix a base point $x_{0} \in N-F$ and identify $\psi^{-1}\left(x_{0}\right)$ with $\{1, \ldots, d\}$. Lifting loops in $N-F$ based at $x_{0}$ then yields a homomorphism $\pi_{1}\left(N-F, x_{0}\right) \rightarrow \Sigma_{d}$. For a loop which runs along a path from $x_{0}$ to a small circle $C$ about $x \in F$, around the circle, and back to $x_{0}$ by the same path one obtains a permutation $\alpha$ whose conjugacy class $A$ is the partition associated to $x$ in the corresponding branched covering. The partition $A=\left[a_{1}, \ldots, a_{r}\right]$ where $\psi^{-1}(C)$ consists of $r$ circles mapped by degrees $a_{1}, \ldots, a_{r}$.

Since one knows the fundamental groups of surfaces (see [9], for example), one may then write down the algebraic conditions for the existence of a connected $d$-fold covering.

LEMmA 2.1. Let $N$ be a closed, connected surface and $\mathscr{D}=\left\{A_{1}, \ldots, A_{k}\right\}$ be a collection of partitions of $d$ (repetitions allowed). Then a necessary and sufficient condition that there exist a $d$-fold branched covering $\phi: M \rightarrow N$ with $M$ closed and connected and with branch data 010 is as follows:

(a) For $N=S^{2}$, there exist elements $\alpha_{i} \in \Sigma_{d}, \alpha_{i} \in A_{i}$, such that $\alpha_{1} \cdots \alpha_{k}=1$ and $\left\langle\alpha_{1}, \ldots, \alpha_{k}\right\rangle$ acts transitively on $\{1, \ldots, d\}$.

(b) For $N=T_{n}$, a connected sum of $n \geqslant 1$ copies of $S^{1} \times S^{1}$, there exist elements $\alpha_{i} \in \Sigma_{d}, \alpha_{i} \in A_{i}$, and $\beta_{j}, \gamma_{j} \in \Sigma_{d}, 1 \leqslant j \leqslant n$, such that

$$
\alpha_{1} \cdots \alpha_{k} \beta_{1} \gamma_{1} \beta_{1}^{-1} \gamma_{1}^{-1} \cdots \beta_{n} \gamma_{n} \beta_{n}^{-1} \gamma_{n}^{-1}=1
$$

and $\left\langle\alpha_{1}, \ldots, \alpha_{k}, \beta_{1}, \gamma_{1}, \ldots, \beta_{n}, \gamma_{n}\right\rangle$ acts transitively on $\{1, \ldots, d\}$.

(c) For $N=U_{n}$, a connected sum of $n \geqslant 1$ projective planes $\mathbf{R} P^{2}$, there exist elements $\alpha_{i} \in \Sigma_{d}, \alpha_{i} \in A_{i}$, and $\beta_{j} \in \Sigma_{d}, 1 \leqslant j \leqslant n$, such that $\alpha_{1} \cdots \alpha_{k} \beta_{1}^{2} \cdots \beta_{n}^{2}=1$ and $\left\langle\alpha_{1}, \ldots, \alpha_{k}, \beta_{1}, \ldots, \beta_{n}\right\rangle$ acts transitively on $\{1, \ldots, d\}$.

When $N=U_{n}, \pi_{1}\left(N-B_{\phi}\right)$ is generated by elements $x_{1}, \ldots, x_{k}, y_{1}, \ldots, y_{n}$ with the relation $x_{1} \cdots x_{k} y_{1}^{2} \cdots y_{n}^{2}=1$, while $\pi_{1}(N)$ is generated by $y_{1}, \ldots, y_{n}$ where $y_{1}^{2}$ $\cdots y_{n}^{2}=1$. The orientable double cover $\tilde{N} \rightarrow N$ corresponds to the subgroup of $\pi_{1}(N)$ which is the kernel of the homomorphism $w: \pi_{1}(N) \rightarrow\{+1,-1\}$ where $w\left(y_{j}\right)=-1$ for $1 \leqslant j \leqslant n$. Similarly the orientable double cover of $N-B_{\phi}$ corresponds to the kernel of $w: \pi_{1}\left(N-B_{\phi}\right) \rightarrow\{+1,-1\}$ where $w\left(x_{i}\right)=+1,1 \leqslant i \leqslant k$, and $w\left(y_{j}\right)=-1,1 \leqslant j \leqslant n$. On the other hand, if $\rho: \pi_{1}\left(N-B_{\phi}\right) \rightarrow \Sigma_{d}$ is the homomorphism with $\rho\left(x_{i}\right)=\alpha_{i}$ and $\rho\left(y_{j}\right)=\beta_{j}$ which determines the branched covering $\phi: M \rightarrow N$ then the covering $M-\phi^{-1} B_{\phi} \rightarrow N-B_{\phi}$ corresponds to the subgroup $\rho^{-1}\left(\Sigma_{d-1}\right) \subset \pi_{1}\left(N-B_{\phi}\right)$. Thus one has the following characterization of orientability.

LeMMA 2.2. If $\phi: M \rightarrow N$ is a connected, degree $d$ branched covering of $N=U_{n}$, then $M$ is orientable if and only if $\rho^{-1}\left(\Sigma_{d-1}\right) \subset \operatorname{ker}(w)$. 
Proposition 2.3. Let $\phi: M \rightarrow N$ be a degree $d$ branched covering of closed, connected surfaces. If $N$ is orientable, then so is $M$; if $d$ is odd and $N$ nonorientable, then so is $M$.

Proof. The orientability or nonorientability of a surface is not changed by deleting a finite set of points. If $N$ is orientable; then so is $N-B_{\phi}$. An orientation of $N-B_{\phi}$ can be pulled back to an orientation of $M-\phi^{-1} B_{\phi}$. Therefore $M$ is orientable.

Now suppose $d$ is odd and $N$ is nonorientable. Then there is an orientation-reversing simple closed curve $C \subset N-B_{\phi}$. Some component $C_{i}$ of $\phi^{-1}(C)$ maps by odd degree to $C$, and hence is also orientation-reversing in $M$.

Proposition 2.4. If $\phi: M \rightarrow N$ is any degree $d$ branched covering of closed, connected surfaces, then the total branching $v(\phi)$ satisfies $v(\phi)=d \chi(N)-\chi(M)$.

Proof. Triangulate $N$ so that each point of $B_{\phi}$ is a vertex. The homotopy lifting property shows that the edges in $N$ can be lifted to $M$ to induce a triangulation of $M$ with respect to which $\phi$ is simplicial. Over each simplex of $N$ not in $B_{\phi}$ there are exactly $d$ simplices in $M$. If $x \in B_{\phi}$ then $\left|\phi^{-1}(x)\right|=r$ where $A(x)=\left[a_{1}, \ldots, a_{r}\right]$. Using the formula $\chi=V-E+F$ one then computes directly that $\chi(M)=d \chi(N)$ $-\sum_{i=1}^{k}\left(d-r_{i}\right)$, where $B_{\phi}=\left\{x_{1}, \ldots, x_{k}\right\}$ and $A\left(x_{i}\right)=\left[a_{i l}, \ldots, a_{i r_{i}}\right], 1 \leqslant i \leqslant k$. This yields the required formula.

Corollary 2.5. Let $\phi: M \rightarrow N$ be any degree $d$ branched covering of closed, connected surfaces. Then

$$
v(\phi) \geqslant \begin{cases}2 d-2 & \text { if } N=S^{2} \\ d-2 & \text { if } N=\mathbf{R} P^{2} \text { and } M \text { is orientable } \\ d-1 & \text { if } N=\mathbf{R} P^{2} \text { and } M \text { is nonorientable. }\end{cases}
$$

Proof. If $M$ is orientable, then $\chi(M) \leqslant 2=\chi\left(S^{2}\right)$; if $M$ is nonorientable, then $\chi(M) \leqslant 1=\chi\left(\mathbf{R} P^{2}\right)$. Now apply Proposition 2.4 .

Proposition 2.6. If $\phi: M \rightarrow N$ is any degree $d$ branched covering of closed, connected surfaces, then the total branching $v(\phi)$ is even.

Proof. Let $\phi$ have branch data $\mathscr{D}=\left\{A_{1}, \ldots, A_{k}\right\}$ where $A_{i}=\left[a_{i 1}, \ldots, a_{i r_{i}}\right], 1 \leqslant i$ $\leqslant k$. By definition $v(\phi)=\sum_{i=1}^{k}\left(d-r_{i}\right)$. Note that $v\left(A_{i}\right)=d-r_{i}$ can be interpreted as the minimum number of transpositions required to write any $\alpha_{i} \in A_{i}$ as a product of transpositions. Thus $v(\phi)$ is even if and only if the product $\alpha_{1} \cdots \alpha_{k}$ belongs to the alternating group for some (or any) choice of $\alpha_{i} \in A_{i}$. But by Lemma 2.1, $\alpha_{1}, \ldots, \alpha_{k}$ can be chosen so that $\alpha_{1} \cdots \alpha_{k}$ is trivial, a product of commutators, or a product of squares, all of which lie in the alternating group.

Alternate proof of Proposition 2.6. Consider $\mathbf{Z}_{2}$ homology and cohomology. For any closed surface $N, w_{2}(N)=w_{1}(N)^{2}$ and $\chi(N)=\left\langle w_{1}(N)^{2},[N]\right\rangle \bmod 2$, where $[N]$ is the fundamental class and $w_{*}(N) \in H^{*}\left(N ; \mathbf{Z}_{2}\right) \approx$ $\operatorname{Hom}\left(H_{*}\left(N: \mathbf{Z}_{2}\right) ; \mathbf{Z}_{2}\right)$ is the Stiefel-Whitney class. Also the proof of Proposition 2.3 
shows that $\phi^{*} w_{1}(N)=w_{1}(M)$. Then modulo 2

$$
\begin{aligned}
\chi(M) & =\left\langle w_{1}(M)^{2},[M]\right\rangle \\
& =\left\langle\left(\phi^{*} w_{1}(N)\right)^{2},[M]\right\rangle=\left\langle\phi^{*}\left(w_{1}(N)^{2}\right),[M]\right\rangle \\
& =\left\langle w_{1}(N)^{2}, \phi_{*}[M]\right\rangle=\left\langle w_{1}(N)^{2}, d[N]\right\rangle \\
& =d\left\langle w_{1}(N)^{2},[N]\right\rangle=d \chi(N) .
\end{aligned}
$$

Thus $v(\phi)=d \chi(N)-\chi(M) \equiv 0 \bmod 2$.

Technical note. The condition that $v(\phi)=\Sigma v\left(A_{i}\right) \equiv 0 \bmod 2$ is the analogue in dimension 2 of the unusual relation that $\cup\left\{\mathbf{R} P\left(\tilde{\nu}_{i j}\right): d_{i j}\right.$ is even $\} \rightarrow N \times \mathbf{R} P^{\infty}$ bounds for higher-dimensional branched coverings [13, §3]. Here $\tilde{\nu}_{i j}$ represents the normal bundle of a component of $\phi^{-1}\left(B_{\phi}\right)$.

The final result of this section is the statement of a further condition satisfied by an orientable branched covering of a nonorientable surface.

Proposition 2.7. Let $d$ be an even integer, $\mathscr{D}=\left\{A_{1}, \ldots, A_{k}\right\}$ a collection of partitions of $d$, and $N$ be a closed, connected, nonorientable surface. Then a necessary and sufficient condition that there exist a degree $d$ branched covering $\phi: M \rightarrow N$ with branch data $\mathscr{D}$ and $M$ orientable is that for each $i, 1 \leqslant i \leqslant k$, one can express $A_{i}$ as $a$ concatenation $\left[B_{i}, C_{i}\right]$ of two partitions of $d / 2$ such that $\left\{B_{1}, \ldots, B_{k}, C_{1}, \ldots, C_{k}\right\}$ is the branch data of a degree $d / 2$ branched covering of the orientable double covering of $N$.

Proof. Suppose $\phi: M \rightarrow N$ is a degree $d$ branched covering with $M$ orientable and with branch data $\mathscr{D}$. Since $\phi^{*} w_{1}(N)=w_{1}(M)=0$ there is a lift $\tilde{\phi}: M \rightarrow \tilde{N}$ of $\phi$ through the orientable double covering $\pi: \tilde{N} \rightarrow N$. For $x \in B_{\phi}, \pi^{-1}(x)$ consists of two points $x^{\prime}$ and $x^{\prime \prime}$, and $A(x)=\left[A\left(x^{\prime}\right), A\left(x^{\prime \prime}\right)\right]$ where $A\left(x^{\prime}\right)$ and $A\left(x^{\prime \prime}\right)$ are the partitions of $d / 2$ determined by $\tilde{\phi}$.

Conversely suppose $\mathscr{D}=\left\{A_{1}, \ldots, A_{i}\right\}$ and $A_{i}=\left[B_{i}, C_{i}\right], 1 \leqslant i \leqslant k$, is a refinement of $A_{i}$ into two partitions of $d / 2$. Suppose $\theta: M \rightarrow \tilde{N}$ is a degree $d / 2$ branched covering with branch set $\left\{x_{1}^{\prime}, \ldots, x_{k}^{\prime}, x_{1}^{\prime \prime}, \ldots, x_{k}^{\prime \prime}\right\}$ and corresponding branch data $\left\{B_{1}, \ldots, B_{k}, C_{1}, \ldots, C_{k}\right\}$. By composing $\theta$ with an isotopy of $\tilde{N}$ if necessary, one may assume that $\pi\left(x_{i}^{\prime}\right)=\pi\left(x_{i}^{\prime \prime}\right)$. Then $\pi \cdot \theta$ is a branched covering with branch data $\mathscr{D}$.

Note. While pairs of elements in the branch data of $\phi: M \rightarrow \tilde{N}$ may be combined arbitrarily to give the branch data of a branched covering $M \rightarrow N$, it is false that every refinement of the branch data of a $\phi: M \rightarrow N$ is the branch data for a branched covering $M \rightarrow \tilde{N}$. See $\S 5$.

It should be noted that just $N$, the total branching $v$, and the orientability of $M$ always determine at least one branched covering.

Proposition 2.8. Let $M$ and $N$ be closed, connected surfaces and $d \geqslant 2$. Suppose that

(a) $M$ is orientable if $N$ is orientable,

(b) if $d$ is odd or $d=2$ and $N$ is nonorientable, then $M$ is nonorientable.

Then there is some degree d branched covering $\phi: M \rightarrow N$ if and only if $\chi(M) \leqslant d \chi(N)$ and $v=d \chi(N)-\chi(M)$ is even. 
Proof. By the preceding results of this section the given conditions are necessary. For sufficiency choose $\mathscr{D}=\left\{A_{1}, \ldots, A_{k}\right\}$ with $v\left(A_{i}\right)=1$ (so $k=v$ ) i.e. $A_{i}=$ $[2,1, \ldots, 1], 1 \leqslant i \leqslant k$. To see that this data can always be realized, one applies Lemma 2.1 as follows.

First suppose $N=S^{2}$. Then $k=v \geqslant 2 d-2$. Realize $\mathscr{D}$ by the transpositions

$$
(1,2),(1,2),(1,3),(1,3), \ldots,(1, d),(1, d),(1,2),(1,2), \ldots,(1,2),(1.2) \text {. }
$$

Next suppose $N=T_{n}, n \geqslant 1$. Let $\alpha_{1}=\cdots=\alpha_{k}=(1,2), \beta_{1}=(1,2, \ldots, d)$, and $\gamma_{1}=\beta_{2}=\gamma_{2}=\cdots=\gamma_{n}=1$.

If $N$ is nonorientable and $M$ is orientable one can trivially refine $[2,1, \ldots, 1]$ to $[[2,1, \ldots, 1],[1, \ldots, 1]]$ and apply the orientable case to get a degree $d / 2$ branched covering of the orientable double cover $\tilde{N}$. Proposition 2.7 then yields the required branched covering of $N$.

Finally suppose $M$ and $N$ are nonorientable. If $N=\mathbf{R} P^{2}$ then $k=v \geqslant d-1$. If $d=2$ just set $\alpha_{1}=\cdots=\alpha_{k}=(1,2)$ and $\beta_{1}=1$. If $d \geqslant 3$ and is odd, set $\alpha_{1}=(1,2)$, $\alpha_{2}=(1,3), \ldots, \alpha_{d-1}=(1, d)$ and $\alpha_{i}=(1,2)$ for $i \geqslant d$, and set $\beta_{1}=$ $(1,2, \ldots, d)^{(d-1) / 2}$. If $N=U_{n}, n \geqslant 2$, then set $\alpha_{1}=\cdots=\alpha_{k}=(1,2), \beta_{1}=$ $(1,2, \ldots, d), \beta_{2}=(d, d-1, \ldots, 1)$, and $\beta_{j}=1$ for $j \geqslant 3$. One can use Lemma 2.2 to verify that the corresponding branched covering is nonorientable. (See the proof of Proposition 3.3.)

REMARK. The branched coverings constructed above are called simple branched coverings. Every branched covering of surfaces can be approximated by a simple one, and under relatively mild conditions any two simple branched coverings between the same surfaces are equivalent to one another, i.e., equal after allowing pre- and post- composition with homeomorphisms [1].

3. Elementary realizability results. In this section it is proved that the realizability problem always has an affirmative solution when the target has nonpositive Euler characteristic.

LeMma 3.1. If $\alpha \in \Sigma_{d}$ with $v(\alpha)=d-r$, then for any $t \geqslant 0$ with $r+2 t \leqslant d$ one may express $\alpha$ as the product of a d-cycle and an $(r+2 t)$-cycle.

Proof. One may take

$$
\alpha=\left(1, \ldots, a_{1}\right)\left(a_{1}+1, \ldots, a_{2}\right) \cdots\left(a_{r-1}+1, \ldots, a_{r}\right)
$$

where $1 \leqslant a_{1}<a_{2}<\cdots<a_{r}=d$. Let $\beta=\left(1, a_{1}+1, a_{2}+1, \ldots, a_{r-1}+\right.$ $\left.1, b_{1}, \ldots, b_{2 t}\right)$ where $b_{1}<b_{2}<\cdots<b_{2 t}$ are not in the set $\left\{1, a_{1}+1, \ldots, a_{r-1}+1\right\}$. Then $\gamma=\alpha \beta$ is a $d$-cycle: Let $\beta_{0}=\left(1, a_{1}+1, \ldots, a_{r-1}+1\right)$ and $\beta_{1}=\left(1, b_{1}, \ldots, b_{2 t}\right)$, so $\beta=\beta_{0} \beta_{1}$; then

$$
\begin{aligned}
\alpha \beta= & \alpha \beta_{0} \beta_{1}=(1,2, \ldots, d)\left(1, b_{1}, \ldots, b_{2 t}\right) \\
= & \left(1, \ldots, b_{1}-1, b_{2}, \ldots, b_{3}-1, b_{4}, \ldots, b_{2 t-1}-1,\right. \\
& \left.b_{2 t}, \ldots, d, b_{1}, \ldots, b_{2}-1, b_{3}, \ldots, b_{2 t-2}-1, b_{2 t-1}, \ldots, b_{2 t}-1\right) .
\end{aligned}
$$

Thus $\alpha=\gamma \beta^{-1}$ has the required form. 
COROLlaRY 3.2. Every element in the alternating group on $d$ symbols can be expressed as

(i) a product of two d-cycles,

(ii) a commutator $\alpha \beta \alpha^{-1} \beta^{-1}$ with $\alpha$ a d-cycle,

(iii) a product of two squares $\alpha^{2} \beta^{2}$ with $\alpha \beta$ a d-cycle.

Proof. If $\gamma$ lies in the alternating group $v(\gamma)=d-r$ is even. By Lemma 3.1, $\gamma=\sigma \tau$ where $\sigma$ and $\tau$ are $d$-cycles. Now $\tau$ is conjugate to $\sigma^{-1}$, so $\tau=\beta \sigma^{-1} \beta^{-1}$, so $\gamma=\sigma \beta \sigma^{-1} \beta^{-1}$. Finally $\sigma$ is conjugate to $\tau$ so $\gamma=\left(\alpha \tau \alpha^{-1}\right) \tau=\alpha^{2}\left(\alpha^{-1} \tau\right)^{2}$ for some $\alpha$ and $\alpha\left(\alpha^{-1} \tau\right)=\tau$ is a $d$-cycle.

Proposition 3.3. Let $N$ be a closed, connected surface with Euler characteristic $\chi(N) \leqslant 0$, and let $\mathscr{D}=\left\{A_{1}, \ldots, A_{k}\right\}$ be a collection of partitions of $d$ with $v(\mathscr{D})$ even. Then there is a degree $d$ branched covering $\phi: M \rightarrow N$ with branch data $(2)$ such that $M$ is connected and $M$ is orientable if and only if $N$ is.

Proof. Arbitrarily choose $\alpha_{i} \in A_{i}, 1 \leqslant i \leqslant k$. Then $\alpha_{1} \cdots \alpha_{k}$ lies in the alternating group since $v(\mathscr{D})$ is even.

First consider the case when $N=T_{n}$, an orientable surface of genus $n \geqslant 1$. By Lemma 2.1(b), it suffices to find $\beta_{1}, \gamma_{1}, \ldots, \beta_{n}, \gamma_{n} \in \Sigma_{d}$ such that $\alpha_{1} \cdots \alpha_{k}\left[\beta_{1}, \gamma_{1}\right]$ $\cdots\left[\beta_{n}, \gamma_{n}\right]=1$ and $\left\langle\alpha_{1}, \ldots, \alpha_{k}, \beta_{1}, \gamma_{1}, \ldots, \beta_{n}, \gamma_{n}\right\rangle$ acts transitively. Simply apply Corollary 3.2 to write $\left(\alpha_{1} \cdots \alpha_{k}\right)^{-1}=\beta_{1} \gamma_{1} \beta_{1}^{-1} \gamma_{1}^{-1}$ where $\beta_{1}$ is a $d$-cycle and set $\beta_{i}=\gamma_{i}=1$ for $i>1$. By Proposition 2.3 the total space $M$ of the corresponding branched covering is automatically orientable.

Second, consider the case when $N=U_{n}$, a connected sum of $n \geqslant 2$ projective planes. By Lemma 2.1(c), one must find elements $\beta_{1}, \ldots, \beta_{n} \in \Sigma_{d}$ such that $\alpha_{1}$ $\cdots \alpha_{k} \beta_{1}^{2} \cdots \beta_{n}^{2}=1$ and $\left\langle\alpha_{1}, \ldots, \alpha_{k}, \beta_{1}, \ldots, \beta_{n}\right\rangle$ acts transitively. Apply Corollary 3.2 to write $\left(\alpha_{1} \cdots \alpha_{k}\right)^{-1}=\beta_{1}^{2} \beta_{2}^{2}$ where $\beta_{1} \beta_{2}$ is a $d$-cycle, and set $\beta_{j}=1$ for $j \geqslant 3$. It remains to verify that in this case the total space $M$ of the corresponding branched covering is nonorientable. The orientation homomorphism $w: \pi_{1}\left(U_{n}-B_{\phi}, x_{0}\right) \rightarrow \mathbf{Z}_{2}$ is given by $w\left(a_{i}\right)=+1$ and $w\left(b_{j}\right)=-1$ where $a_{i}, b_{j}$ denote the generators described in $\S 2$ corresponding to $\alpha_{i}, \beta_{j}$ above. Then $w\left(b_{1} b_{2}\right)=+1$ and $\beta_{1} \beta_{2}$ is a $d$-cycle. Therefore there is an $r$ such that $\beta_{1}\left(\beta_{1} \beta_{2}\right)^{r}$ fixes 1 , i.e. lies in $\Sigma_{d-1}$, while $w\left(\beta_{1}\left(\beta_{1} \beta_{2}\right)^{r}\right)=-1$. By Lemma $2.2, M$ is nonorientable.

Proposition 3.4. Let $N$ be a closed, connected, nonorientable surface with $\chi(N) \leqslant 0$, and let $\mathscr{D}=\left\{A_{1}, \ldots, A_{k}\right\}$ be a collection of partitions of an even integer $d$ with $v(\mathscr{D})$ even. Then there is a degree $d$ branched covering $\phi: M \rightarrow N$ with branch data $D$ such that $M$ is connected and orientable if and only if each $A_{i}$ refines $[d / 2, d / 2]$.

Proof. The necessity of the final condition is given by Proposition 2.7. To prove the sufficiency choose a refinement $A_{i}=\left[B_{i}, C_{i}\right], 1 \leqslant i \leqslant k$, where $B_{i}$ and $C_{i}$ are partitions of $d / 2$. Let $\mathcal{E}=\left\{B_{1}, \ldots, B_{k}, C_{1}, \ldots, C_{k}\right\}$. Then $v(\mathcal{E})=v(\mathscr{D})$ is even, and $\chi(\tilde{N})=2 \chi(N) \leqslant 0$, where $\tilde{N}$ is the orientable double cover of $N$. By Proposition 3.3 , there is a degree $d / 2$ branched covering $\psi: M \rightarrow \tilde{N}$ with branch data $\mathcal{E}$ and $M$ connected and orientable. Then by Proposition 2.7 the desired branched covering $\phi$ : $M \rightarrow N$ exists. 
4. Products in symmetric groups. In order to proceed further with a study of realizability of branching data several technical results about products in symmetric groups are required. Recall that $\pi(d)$ denotes the set of all partitions of $d$.

Lemma 4.1. Let $A, B \in \pi(d)$ with $v(A)+v(B)=d-t, t \geqslant 1$. Then for any $\alpha \in A, \beta \in B, v(\alpha \beta) \leqslant d-t$.

Proof. Let $\alpha \in A, \beta \in B$ be representatives and set $\gamma=\alpha \beta$. Suppose $\langle\alpha, \beta\rangle$ acts on $\{1,2, \ldots, d\}$ with exactly $q$ orbits. Since $\gamma \in\langle\alpha, \beta\rangle, \gamma$ must have at least $q$ oribts, i.e. $v(\gamma) \leqslant d-q$. Let $C$ denote the partition determined by $\gamma$. Then $v(A)+v(B)+$ $v(C) \leqslant 2 d-t-q$.

Now $\alpha \beta \gamma^{-1}=1$ so there is a branched covering $\phi: M \rightarrow S^{2}$ with branching data $\sigma_{D}=\{A, B, C\}$, where $M$ is a closed orientable surface with exactly $q$ components. The Hurwitz formula says $d \chi\left(S^{2}\right)-[v(A)+v(B)+v(C)]=\chi(M) \leqslant q \chi\left(S^{2}\right)=$ $2 q$. Therefore $2 d-2 q \leqslant v(A)+v(B)+v(C)$. This means $q \geqslant t$, and therefore $v(\gamma) \leqslant d-q \leqslant d-t$, as required.

Lemma 4.2. Let $A, B \in \pi(d)$ with $v(A)+v(B)=d-t, t \geqslant 1$. Then there exists $\alpha \in A$ and $\beta \in B$ such that

(i) the group $\langle\alpha, \beta\rangle$ generated by $\alpha$ and $\beta$ acts with precisely $t$ orbits; and

(ii) if $C$ is the partition of $\{1, \ldots, d\}$ into the orbits of the action of $\langle\alpha, \beta\rangle$, then $\alpha \beta \in C$.

Proof. First we observe that the second assertion follows from the first. By Lemma 4.1, $v(\alpha \beta) \leqslant d-t$. As in the proof of Lemma 4.1, $2 d-2 t \leqslant v(\alpha)+v(\beta)$ $+v(\alpha \beta) \leqslant 2 d-2 t$. Therefore $v(\alpha \beta)=d-t$. Since the partition associated with $\alpha \beta$ must refine the partition $C$ and $v(\alpha \beta)=v(C)$, it follows that $\alpha \beta \in C$.

The first assertion will be proved by induction on $d$.

If $v(A)=0$, then $\alpha \in A$ must be the identity, so that $\langle\alpha, \beta\rangle=\langle\beta\rangle$ has $d-v(B)$ orbits, as does $\beta \in B$. This same observation applies if $v(B)=0$. This implies the assertion for $d=1(v(A)=v(B)=0)$ and for $d=2(v(A)+v(B) \leqslant 1)$.

Proceeding inductively, suppose $v(A)=d-r>0$. One may then fix a standard representative

$$
\alpha=\left(1,2, \ldots, d_{1}\right)\left(d_{1}+1, \ldots, d_{2}\right) \cdots\left(d_{r-1}+1, \ldots, d_{r}\right) \in A
$$

where $d_{r}=d$ and $d_{1}>1$. Let $B=\left[b_{1}, b_{2}, \ldots, b_{s}\right]$, so that $v(B)=d-s$ and $t=r+$ $s-d$. Represent the unknown $\beta \in B$ as a product $\beta=\beta_{1} \cdots \beta_{s}$ of disjoint cycles, when $\beta_{j}$ represents the $b_{j}$-cycle whose entries remain to be determined.

Observe that $b_{1} \leqslant r$, since $d-1 \geqslant v(A)+v(B) \geqslant(d-r)+\left(b_{1}-1\right)$. Set $\beta_{1}=$ $\left(1, d_{1}+1, \ldots, d_{b_{1}-1}+1\right)$. The orbits of $\langle\alpha\rangle$ acting on $\{1, \ldots, d\}$ are the sets $P_{i}=\left\{d_{i-1}+1, \ldots, d_{i}\right\}$; and with this choice of $\beta_{1}, P_{1} \cup \cdots \cup P_{b_{1}}$ will all lie inside one orbit of $\langle\alpha, \beta\rangle$.

The $d-b_{1}$ points $\{1, \ldots, d\}-\operatorname{supp}\left(\beta_{1}\right)$ not yet assigned to the cycles $\beta_{2}, \ldots, \beta_{s}$ can be partitioned into $r-b_{1}+1$ sets $Q_{1}, \ldots, Q_{r-b_{1}+1}$, where $Q_{1}=\left\{1, \ldots, d_{b_{1}}\right\}-$ $\operatorname{supp}\left(\beta_{1}\right), Q_{2}=P_{h_{1}+1}, \ldots, Q_{r-b_{1}+1}=P_{r}$. Note that $Q_{1} \neq \varnothing$, since $d_{1}>1$. 
Now set $A^{\prime}=\left[\left|Q_{1}\right|, \ldots,\left|Q_{r-b_{1}+1}\right|\right]$ and $B^{\prime}=\left[b_{2}, \ldots, b_{s}\right]$ in $\pi\left(d-b_{1}\right)$, so $v\left(A^{\prime}\right)=$ $\left(d-b_{1}\right)-\left(r-b_{1}+1\right)=d-r-1$ and $v\left(B^{\prime}\right)=\left(d-b_{1}\right)-(s-1)$. Thus $v\left(A^{\prime}\right)$ $+v\left(B^{\prime}\right)=\left(d-b_{1}\right)+d-r-s=\left(d-b_{1}\right)-t$. Let $\alpha^{\prime}=\alpha_{1}^{\prime} \cdots \alpha_{r-b_{1}+1}^{\prime} \in A^{\prime}$ where the entries of $\alpha_{i}^{\prime}$ come from $Q_{i}$. By induction there exists $\beta^{\prime} \in B^{\prime}$ with entries in $\{1, \ldots, d\}-\operatorname{supp}\left(\beta_{1}\right)$ such that $\left\langle\alpha^{\prime}, \beta^{\prime}\right\rangle$ has $t^{\prime}$ orbits where $t^{\prime}=\left(d-b_{1}\right)-$ $v\left(A^{\prime}\right)-v\left(B^{\prime}\right)=t$. Then just set $\beta=\beta_{1} \beta^{\prime}$ to complete the proof.

REMARK. In the situation of Lemma 4.2 it is difficult to prescribe the particular conjugacy type of $\alpha \beta$. If one assumes $v(A) \leqslant v(B)$ and $A=\left[a_{1}, \ldots, a_{r}\right], a_{1} \geqslant \cdots \geqslant$ $a_{r}$, and $B=\left[b_{1}, \ldots, b_{s}\right], b_{1} \geqslant \cdots \geqslant b_{s}$, then examination of the proof shows that one can achieve $\|\boldsymbol{\alpha} \beta\|=\left[\sum_{i=1}^{v(B)} a_{i}, b_{s-t+2}, \ldots, b_{s}\right]$.

Lemma 4.3. Let $A, B \in \pi(d)$ with $v(A)+v(B)=(d-1)+r, r>0$. Then for each $k$ satisfying $0 \leqslant k \leqslant r, k \equiv r \bmod 2$, one may choose $\alpha \in A, \beta \in B$ so that $\langle\alpha, \beta\rangle$ acts transitively on $\{1, \ldots, d\}$ and so that $v(\alpha \beta)=(d-1)-k$.

Proof. Let $B=\left[b_{1}, \ldots, b_{s}\right]$ and $k=r-2 j$. Let $i \geqslant 0$ be determined by the requirement that $v(A)+\left(b_{1}-1\right)+\cdots+\left(b_{i}-1\right) \leqslant d-1$ and $v(A)+\left(b_{1}-1\right)$ $+\cdots+\left(b_{i+1}-1\right)>d-1$. Therefore there is a positive integer $f$ such that $f-1<$ $b_{i+1}-1$ and $v(A)+\left(b_{1}-1\right)+\cdots+\left(b_{i}-1\right)+(f-1)=d-1$. Let $B^{\prime}=$ $\left[b_{1}, \ldots, b_{i}, f, 1, \ldots, 1\right]$ be the induced partition of $d$, and $B^{\prime \prime}=\left[b_{i+1}-f+\right.$ $\left.1, b_{i+2}, \ldots, b_{s}\right]$.

We view $B^{\prime \prime}$ as a partition of $n$ symbols $\left\{u_{1}, \ldots, u_{n}\right\}$, one of which occurs in the $f$-cycle of $\beta^{\prime}$ and otherwise disjoint from the symbols which $\beta^{\prime}$ involves.

By choice $v\left(B^{\prime \prime}\right)=r$ and by Lemma $3.1, B^{\prime \prime}$ can be realized by a product of an $n$-cycle and an $(n-r+2 j)$-cycle in the symbols $\left\{u_{1}, \ldots, u_{n}\right\}$. Equivalently there is $\beta^{\prime \prime} \in B^{\prime \prime}$ and an $n$-cycle $\gamma$ such that $\gamma \beta^{\prime \prime} \in[(n-r+2 j), 1, \ldots, 1]$. We may assume that the $d$-cycle $\alpha \beta^{\prime}$ has the form $\left(u_{1}, \ldots, w_{1}, u_{2}, \ldots, w_{2}, \ldots, u_{n}, \ldots, w_{n}\right)$ and that $\gamma=\left(u_{1}, \ldots, u_{n}\right)$.

Set $\beta=\beta^{\prime} \beta^{\prime \prime}$. The product $\alpha \beta=\left(\alpha \beta^{\prime}\right) \beta^{\prime \prime}$ can then be described as being obtained from $\left(u_{1}, \ldots, u_{n}\right) \beta^{\prime \prime} \in[(n-r+2 j), 1, \ldots, 1]$ by replacing each $u_{j}$ by the block $u_{j}, \ldots, w_{j}$ occurring in $\alpha \beta^{\prime}$. In other words $\alpha \beta$ is the product of $r-2 j+1$ disjoint cycles, so that $v(\alpha \beta)=d-(r-2 j+1)=(d-1)-r+2 j$, as required.

Corollary 4.4. Let $A, B \in \pi(d)$ with $v(A)+v(B) \geqslant d-1$ and $v(A)+v(B) \equiv$ $d+1 \bmod 2$. Then there exist $\alpha \in A, \beta \in B$ such that $\langle\alpha, \beta\rangle$ acts transitively on $\{1, \ldots, d\}$ and so that $\alpha \beta$ is a d-cycle.

Proof. If $v(A)+v(B)=d-1$, this follows from Lemma 4.2. If $v(A)+v(B) \geqslant$ $d+1$, take $k=0$ in Lemma 4.3.

REMARK. Again, in general, it is very difficult to control the sizes of the blocks $u_{j}, \ldots$, occurring in $\alpha \beta^{\prime}$, so that one has trouble controlling the lengths of the cycles in $\alpha \beta$. We shall need a precise result, however, when $v(A)+v(B) \equiv d \bmod 2$.

Lemma 4.5. Let $A, B \in \pi(d)$ with $v(A)+v(B)=d+2 q, q \geqslant 0$. Then there exist $\alpha \in A, \beta \in B$ such that $\langle\alpha, \beta\rangle$ acts transitively on $\{1, \ldots, d\}$ and $\alpha \beta \in[d / 2, d / 2]$ if $A=B=[2, \ldots, 2]$ and $\alpha \beta \in[d-1,1]$ otherwise. 
Proof. First suppose $A=B=[2, \ldots, 2]$ with $d=2 r$. If $\alpha=(1,2)(3,4) \cdots(2 r-$ $1, r)$ and $\beta=(2,3)(4,5) \cdots(2 r-2,2 r-1)(2 r, 1)$, then

$$
\alpha \beta=(1,3, \ldots,(2 r-1))(2 r, 2 r-2, \ldots, 2) \in[r, r] .
$$

Now suppose that not both $A$ and $B$ are $[2, \ldots, 2]$. Then one of $A$ and $B$ contains a term greater than 2 , since otherwise $v(A)+v(B)<d$. Without loss of generality one may assume that $A$ has an entry greater than 2 , since $\alpha \beta \in C$ if and only if $\beta^{-1} \alpha^{-1} \in C$. Let $\alpha=\left(1, \ldots, d_{1}\right)\left(d_{1}+1, \ldots, d_{2}\right) \cdots\left(d_{r-1}+1, \ldots, d_{r}\right) \in A$, with $d_{r}$ $=d$ and $d_{1}>2$. Since $v(A) \leqslant d-1, v(B)>0$. Therefore $B=\left[b_{1}, \ldots, b_{s}\right]$ with $b_{1} \geqslant 2$.

Consider the partitions $A^{\prime}=\left[a_{1}-1, a_{2}, \ldots, a_{r}\right]$ and $B^{\prime}=\left[b_{1}-1, b_{2}, \ldots, b_{s}\right]$ (where $a_{i+1}=d_{i+1}-d_{i}$ ). Realize $A^{\prime}$ by $\alpha^{\prime}=\left(1,2, \ldots,\left(d_{1}-1\right)\right)\left(d_{1}+1, \ldots, d_{2}\right)$ $\cdots\left(d_{r-1}+1, \ldots, d_{r}\right)$. Here we are viewing $A^{\prime}$ and $B^{\prime}$ as partitions of $\{1, \ldots, d\}-$ $\left\{d_{1}\right\}$. Note that

$$
v\left(A^{\prime}\right)+v\left(B^{\prime}\right)=(v(A)-1)+(v(B)-1)=(d-1)-1+2 q .
$$

By Lemma 4.2 there is $\beta^{\prime} \in B^{\prime}$ such that $\alpha^{\prime} \beta^{\prime}$ is a $(d-1)$-cycle. Referring to the actual construction of $\beta^{\prime}$ in the proof of Lemma 4.2, one may take $\beta^{\prime}$ to begin with the $\left(b_{1}-1\right)$-cycle $\left(1, d_{1}+1, \ldots, d_{b_{1}-2}+1\right)$.

Now $\alpha=\alpha^{\prime}\left(1, d_{1}\right)$. Set $\beta=\left(1, d_{1}\right) \beta^{\prime}$. Then $\beta \in B$ and $\alpha \beta$ is a $(d-1)$-cycle. Moreover, $\langle\alpha, \beta\rangle$ is transitive on $\{1, \ldots, d\}$ since $\alpha$ moves $d_{1}$, while $\alpha \beta$ is a $(d-1)$-cycle on the symbols different from $d_{1}$.

RemarK. If $A=B=[2, \ldots, 2]$ with $d=2 r$ and $\alpha \in A, \beta \in B$ such that $\langle\alpha, \beta\rangle$ is transitive, then $\alpha \beta \in[r, r]$ necessarily. One may invoke transitivity appropriately to see that $\alpha$ and $\beta$ may be labelled so as to have the precise form used in the beginning of the proof of Lemma 4.5. This observation leads to a simple family of nonrealizable branching data: $\{[2, \ldots, 2],[2, \ldots, 2],[1, d-1]\}$. See also Corollary 6.4 for another look at data of this type.

Corollary 4.4 and Lemma 4.5 also follow from [2].

5. Branched coverings of the sphere and projective plane. The object of this section is to apply the facts obtained in $\$ 4$ about products in symmetric groups to the problems of realizing branch data for branched coverings of $\mathbf{R} P^{2}$ and $S^{2}$.

THEOREM 5.1. Let $\mathscr{D}=\left\{A_{1}, \ldots, A_{k}\right\}$ be a collection of partitions of a positive integer $d$. Then there is a branched covering $M \rightarrow \mathbf{R} P^{2}$ of degree $d$, with $M$ connected, and with branch data $\mathcal{D}$ if and only if $\Sigma v\left(A_{i}\right) \geqslant d-1$ and $\Sigma v\left(A_{i}\right)$ is even. Moreover $M$ can be chosen to be nonorientable.

Proof. The necessity of the conditions on $\Sigma v\left(A_{i}\right)$ is given by Corollary 2.5 and Proposition 2.6. For sufficiency one may repeatedly apply Lemmas 4.2, 4.3 and 4.5 to choose representative permutations $\alpha_{i} \in A_{i}, 1 \leqslant i \leqslant k$, so that $\left\langle\alpha_{1}, \ldots, \alpha_{k}\right\rangle$ acts transitively on $\{1, \ldots, d\}$ and $\alpha_{1} \cdots \alpha_{k} \in[d]$ if $d$ is odd, while $\alpha_{1} \cdots \alpha_{k} \in[d-1,1]$ or $\alpha_{1} \cdots \alpha_{k} \in[d / 2, d / 2]$ if $d$ is even.

If $d$ is odd, each $d$-cycle is a square in $\Sigma_{d}$, so there is a $d$-cycle $\beta$ such that $\alpha_{1} \cdots \alpha_{k} \beta^{2}=1$. If $d$ is even, and $\alpha_{1} \cdots \alpha_{k} \in[d-1,1]$, then there is $\beta \in[d-1,1]$ 
such that $\alpha_{1} \cdots \alpha_{k} \beta^{2}=1$; if $\alpha_{1} \cdots \alpha_{k} \in[d / 2, d / 2]$, then there is $\beta \in[d]$ such that $\alpha_{1} \cdots \alpha_{k} \beta^{2}=1$.

In each case by Lemma 2.1 , there is a branched covering $M \rightarrow \mathbf{R} P^{2}$ with branch data $\mathscr{Q}$. To see that $M$ is nonorientable, note that $\left\langle\alpha_{1}, \ldots, \alpha_{k}\right\rangle$ is transitive, so that there is $\gamma \in\left\langle\alpha_{1}, \ldots, \alpha_{k}\right\rangle$ with $\gamma(\beta(1))=1$. Then $\gamma \beta \in \Sigma_{d-1}$ and $w_{1}(\gamma \beta)=$ $w_{1}(\gamma) w_{1}(\beta)=1 \cdot(-1)=-1$, so that $M$ is nonorientable by Lemma 2.2 .

We now turn to the situation of branched coverings of $S^{2}$. At the end of this section we address the question of orientable branched coverings of $\mathbf{R} P^{2}$.

If $\mathscr{Q}=\left\{A_{1}, \ldots, A_{k}\right\}$ is a collection of partitions of $\mathscr{D}$ we say simply that $\mathscr{D}$ is realizable if $\mathscr{D}$ arises as the branch data of a connected branched covering of $S^{2}$. By Lemma 2.1 this is equivalent to the existence of representatives $\alpha_{i} \in A_{i}$ such that $\alpha_{1} \cdots \alpha_{k}=1$ and $\left\langle\alpha_{1}, \ldots, \alpha_{k}\right\rangle$ is transitive on $\{1, \ldots, d\}$.

Proposition 5.2. A collection $\mathscr{D}=\left\{A_{1}, \ldots, A_{k}\right\}$ of partitions of $d$, with some $A_{i}=[d]$ is realizable if and only if $v(\mathscr{D}) \geqslant 2 d-2$ and $v(\mathscr{D}) \equiv 0 \bmod 2$.

Proof. It remains only to prove sufficiency. If $k=2$, the hypothesis implies $A_{1}=A_{2}=[d]$ and one lets $\alpha_{1}=(1, \ldots, . d)$ and $\alpha_{2}=\alpha_{1}^{-1}$. If $k=3$ this is Corollary 4.4. Now proceed inductively to consider the case $k>3$.

We may assume $A_{k}=[d]$. Consider two subcases. First suppose some $v\left(A_{i}\right)+$ $v\left(A_{j}\right) \leqslant d-1(i \neq j)$; say $v\left(A_{1}\right)+v\left(A_{2}\right) \leqslant d-1$. By Lemma 4.2 there exist $\alpha_{1} \in A_{1}, \alpha_{2} \in A_{2}$ such that $v\left(\alpha_{1} \alpha_{2}\right)=v\left(\alpha_{1}\right)+v\left(\alpha_{2}\right)$. Let $A=\left\|\alpha_{1} \alpha_{2}\right\|$. Then ' 12 ' $=$ $\left\{A, A_{3}, \ldots, A_{k}\right\}$ satisfies the hypotheses of the proposition. Thus there exist $\alpha \in A$, $\alpha_{i} \in A_{i}, 3 \leqslant i \leqslant k$, which realize $\mathscr{D}^{\prime}$. Now $\alpha$ is conjugate to $\alpha_{1} \alpha_{2}$, say $\alpha=\gamma \alpha_{1} \alpha_{2} \gamma^{-1}$. Then $\gamma \alpha_{1} \gamma^{-1}, \gamma \alpha_{2} \gamma^{-1}, \alpha_{3}, \ldots, \alpha_{k}$ realize $\mathscr{D}$.

For the second subcase suppose $v\left(A_{i}\right)+v\left(A_{j}\right) \geqslant d$ for all $i \neq j$. By Lemmas 4.3 and 4.5 there exist $\alpha_{1} \in A_{1}, \alpha_{2} \in A_{2}$ such that $v\left(\alpha_{1} \alpha_{2}\right) \geqslant d-2$, and, of course, $v\left(\alpha_{1} \alpha_{2}\right) \equiv v\left(\alpha_{1}\right)+v\left(\alpha_{2}\right) \bmod 2$. Again set $\mathscr{D}^{\prime}=\left\{A, A_{3}, \ldots, A_{k}\right\}$ where $A=\left\|\alpha_{1} \alpha_{2}\right\|$. Then $v\left(\mathcal{O}^{\prime}\right) \equiv 0 \bmod 2$ and $\left.v\left({ }^{\prime}\right)^{\prime}\right) \geqslant(d-2)+d / 2+(d-1) \geqslant 2 d-2$. Thus ' $(1)$ ' is realizable by induction. As before this implies that $(1)$ is realizable.

Proposition 5.3. A collection of partitions of $d$, $(D)=\left\{A_{1}, \ldots, A_{k}\right\}$, with some $A_{i}=[d-1,1]$, is realizable if and only if $v(\mathscr{D}) \geqslant 2 d-2, v(\cup 1) \equiv 0 \bmod 2$, and $(1)$ is not

(1) $\{[2,2], \ldots,[2,2],[3,1]\}(d=4, k \geqslant 3)$ or

(2) $\{[2, \ldots, 2],[2, \ldots, 2],[d-1,1]\}(d=2 r, k=3)$.

Proof. It was observed in $\$ 4$ that the data (2) is not realizable. That data (1) is not realizable follows from the fact that the permutations in $[2,2]$ generate the 2-Sylow subgroup of $\Sigma_{4}$ which contains no 3-cycle.

It remains to prove the realizability of the remaining allowable data 12 . The hypotheses imply $k \geqslant 3$; when $k=3$ it is a consequence of Lemma 4.5. Inductively consider the case $k \geqslant 4$. It may be assumed that $A_{k}=[d-1,1]$. A reduction procedure similar to that used in the proof Proposition 5.2 will be employed.

By Lemmas 4.2, 4.3 and 4.5 there exist $\alpha_{1} \in A_{1}$ and $\alpha_{2} \in A_{2}$ such that either $v\left(\alpha_{1} \alpha_{2}\right)=v\left(\alpha_{1}\right)+v\left(\alpha_{2}\right)$ or $v\left(\alpha_{1} \alpha_{2}\right) \geqslant d-2$ (and, of course, $v\left(\alpha_{1} \alpha_{2}\right) \equiv v\left(\alpha_{1}\right)+$ $\left.v\left(\alpha_{2}\right) \bmod 2\right)$. If $d=4$, it may be arranged that $\alpha_{1} \alpha_{2} \notin[2,2]$, since $\mathscr{D}$ does not have 


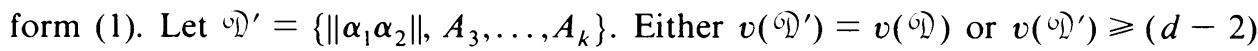
$+1+(3-2)=2 d-3$. Since $v\left(\mathscr{D}^{\prime}\right) \equiv v(\mathscr{D}) \bmod 2$, in fact $v\left(\mathscr{D}^{\prime}\right) \geqslant 2 d-2$. By induction ${ }^{\circ} \mathcal{D}^{\prime}$ is realizable; as in the proof of Proposition 5.2, $\mathcal{Q}$ is then realizable.

TheOREM 5.4. A collection $\mathcal{Q}=\left\{A_{1}, \ldots, A_{k}\right\}$ of partitions of an integer $d \geqslant 2$, $d \neq 4$, is realizable provided $v(\mathcal{O}) \equiv 0 \bmod 2$ and $v(\mathcal{D}) \geqslant 3(d-1)$.

Proof. The cases where $d=2$ are trivial, so assume throughout that $d \geqslant 3$. The case $k \leqslant 2$ is vacuous. If $k=3$, then the hypotheses imply that $A_{1}=A_{2}=A_{3}=[d]$, and that $d$ is odd. In this case simply take $\alpha_{1}=\alpha_{2}=(1,2, \ldots, d)$ and $\alpha_{3}=\left(\alpha_{1} \alpha_{2}\right)^{-1}$. Inductively proceed to the case $k \geqslant 4$.

There will be two cases. First suppose some $v\left(A_{i}\right)+v\left(A_{j}\right) \leqslant d-1, i \neq j$. It may be assumed that $v\left(A_{1}\right)+v\left(A_{2}\right) \leqslant d-1$. By Lemma 4.2 there exist $\alpha_{1} \in A_{1}$ and $\alpha_{2} \in A_{2}$ such that $v\left(\alpha_{1} \alpha_{2}\right)=v\left(\alpha_{1}\right)+v\left(\alpha_{2}\right)$. Let $A=\left\|\alpha_{1} \alpha_{2}\right\|$ and $\mathscr{D}=$ $\left\{A, A_{3}, \ldots, A_{k}\right\}$. By induction ${ }^{\circ} \mathrm{D}^{\prime}$ is realizable. Altering the choice of $\alpha_{1}$ and $\alpha_{2}$ by a conjugation shows that $\mathscr{D}$ is realizable.

Secondly, suppose that $v\left(A_{i}\right)+v\left(A_{j}\right) \geqslant d$ for all $i \neq j$. By Proposition 5.2 it may be assumed that each $v\left(A_{i}\right) \leqslant d-2$. There will be two subcases. First suppose that some $A_{i} \neq[2, \ldots, 2]$. It may be assumed that $A_{1} \neq[2, \ldots, 2]$ and $v\left(A_{1}\right)$ is minimal among $v\left(A_{i}\right)$ where $A_{i} \neq[2, \ldots, 2]$. By Lemmas 4.3 and 4.5 there exist $\alpha_{1} \in A_{1}$ and $\alpha_{2} \in A_{2}$ such that $\alpha_{1} \alpha_{2} \in[d]$ or $[d-1,1]$. Let $A=\left\|\alpha_{1} \alpha_{2}\right\|$ and $\mathscr{D}^{\prime}=$ $\left\{A, A_{3}, \ldots, A_{k}\right\}$. Now $\left.v\left({ }^{(\mathcal{D}}\right)^{\prime}\right) \geqslant(d-2)+(k-2) d / 2 \geqslant 2 d-2$, since in $\mathcal{D}$ at most one $v\left(A_{i}\right) \leqslant d / 2$ and $k \geqslant 4$. Now O $)^{\prime}$ is realizable except if $k=4$ and $A_{3}=A_{4}=$ $[2, \ldots, 2]$; but then $v\left(\mathcal{O}_{2}\right) \leqslant 2(d-2)+2 d / 2=3 d-4<3(d-1)$, contrary to assumption. Altering the choice of $\alpha_{1}$ and $\alpha_{2}$ by an appropriate conjugation then realizes 'DI.

It remains to suppose $A_{i}=[2, \ldots, 2]$ for all $i$. Then $v(\mathscr{D})=k d / 2>3(d-1)$ since $v(\cdot D) \equiv 0 \bmod 2 ;$ and thus

$$
k>6(d-1) / d \geqslant 5
$$

because $d$ is even and $d \geqslant 6$. Applying Lemma 4.5 twice one realizes $\alpha_{1} \in A_{1}$, $\alpha_{2} \in A_{2}, \alpha_{3} \in A$, such that $\alpha_{1} \alpha_{2} \alpha_{3} \in[d]$ or $[d-1,1]$. Since $k \geqslant 6$, Propositions 5.2 and 5.3 then imply that the reduced data $\left\{\left\|\alpha_{1} \alpha_{2} \alpha_{3}\right\|, A_{4}, \ldots, A_{k}\right\}$ is realizable; hence so is (D).

REMARK. With care one can refine this proof to somewhat improve the bound $n(d)=3(d-1)$. For example if $d$ is odd, the difficulties with $[2, \ldots, 2]$ do not arise and one can get by with $n(d)=3 d-5$.

REMARK. The method of proof used for Theorem 5.4 applies to other general families of data $\mathscr{D}=\left\{A_{1}, \ldots, A_{k}\right\}$ as follows. We say that $\mathscr{D}$ is splittable if there is a permutation $\sigma$ of $\{1, \ldots, k\}$ and an integer $s$ such that $\sum_{i=1}^{s} v\left(A_{\sigma(i)}\right) \geqslant d-1$ and $\sum_{i=s+1}^{k} v\left(A_{\sigma(i)}\right) \geqslant d-1$. One may as well assume $\sigma$ is the identity. Assuming $v(\mathscr{D}) \equiv 0 \bmod 2$, one can then find $\alpha_{i} \in A_{i}, 1 \leqslant i \leqslant k$, such that $\alpha_{1} \cdots \alpha_{s}$ and $\alpha_{s+1} \cdots \alpha_{k}$ are both $d$-cycles or both $(d-1)$-cycles. An appropriate conjugation then shows $\mathscr{D}$ is realizable. 
REMARK. The problem of realizing branch data $\mathscr{D}$ with $v(\mathscr{D})=2(d-1)$ corresponds to the problem of realizing rational functions $\mathbf{C} \cup\{\infty\} \rightarrow \mathbf{C} \cup\{\infty\}$ with prescribed multiplicities for the zeros and poles. The problem of realizing polynomial maps of degree $d, \mathbf{C} \rightarrow \mathbf{C}$, with prescribed multiplicities for its zeros corresponds to the problem of realizing data $\mathscr{D})$ with $v(\mathscr{D})=2(d-1)$ such that $\left.{ }_{1}\right)$ contains $[d]$. As we have seen the latter realizability problem has a complete affirmative solution. Thom in [14] gives another solution for this special case.

COROLLARY 5.5. If $d \neq 4$ there exist only a finite number of nonrealizable sets of branch data of degree $d$.

Proof. Only finitely many sets of branch data $\mathscr{D}$ satisfy $v(\mathscr{D})<3(d-1)$.

COMPLEMENT 5.6. For $d=4$ a set ${ }^{\circ} D=\left\{A_{1}, \ldots, A_{k}\right\}$ of branch data is realizable if and only if $v(\mathcal{D}) \geqslant 2 d-2=6, \quad v(\mathcal{Q}) \equiv 0 \bmod 2$, and $\mathcal{Q}$ is not $\{[2,2],[2,2], \ldots,[2,2],[3,1]\}$.

Proof. It was observed in $\S 4$ that the listed data cannot be realized. Suppose $\mathscr{D}$ is any other allowable set of branch data. If $\mathcal{D})$ contains [4] or $[3,1]$, then realizability follows from Proposition 5.2 or 5.3. Otherwise the entries of $\mathscr{D}$ are [2, 2]'s and/or $[2,1,1]$ 's. If both appear, the now standard reduction procedure reduces one to the case where [4] occurs. If only [2, 1, 1] appears (at least 6 times), then 3 entries can be combined to reduce one to the [4] case. If only [2,2] appears, one again reduces to the case of fewer entries by combining two [2,2]'s into one.

When $d$ is not prime there always exist nonrealizable branch data.

Proposition 5.7. If $d=a b, a, b>1$, then

$$
\text { 이 }=\{[a, \ldots, a],[b+1,1, \ldots, 1],[a, a(b-1)]\}
$$

satisfies $v($ (ㄱ) $)=2 d-2$, but is nonrealizable.

Proof. The plan of proof is to fix $\beta=(1,2, \ldots, b+1) \in[b+1,1, \ldots, 1]$ and to consider all possible $\alpha \in[a, \ldots, a]$, with $\langle\alpha, \beta\rangle$ transitive, and see that $\alpha \beta$ cannot lie in $[a, a(b-1)]$.

Transitivity requires that none of the $b a$-cycles of $\alpha$ can involve only entries greater than $b+1$. It follows that $(b-1) a$-cycles each contain exactly one entry less than or equal to $b+1$ and one of them contains exactly two such entries. So without loss one can assume $\alpha$ has the form

$$
(1 \cdots j \cdots)(2 \cdots) \cdots(j-1, \ldots)(j+1, \ldots) \cdots(b+1, \ldots)
$$

for some $j \leqslant b+1$.

Thus

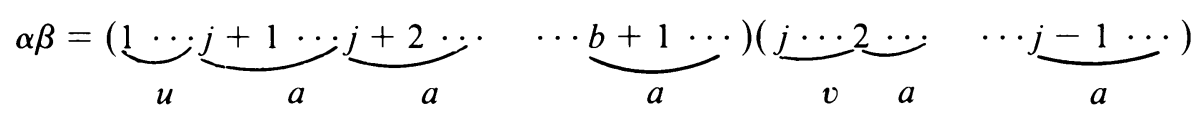

where $u+v=a, u, v \geqslant 1$. Thus $\alpha \beta \in[x, d-x]$ where $x \equiv u \bmod a$ and $d-x \equiv$ $v \bmod a$. In particular, neither $x$ nor $d-x$ is divisible by $a$, and therefore $\alpha \beta=$ $\notin[a, a(b-1)]$. 
REMARK. It is a problem of some interest to determine just what branch data is realizable. One would like to determine better bounds $n(d)$ such that $v(\mathcal{D}) \geqslant n(d)$ implies $\mathscr{D}$ is realizable. One would also like to decide whether all allowable data $(D)$ is realizable when $d$ is prime. The reduction techniques of this section reduce the latter question to the case of data with just 3 entries. Hand and computer checks have shown that the answer is yes for $d=3,5,7$. Already for $d=11$ large numbers of computations are required.

We now return to the problem of realizing branch data by orientable branched coverings of $\mathbf{R} P^{2}$.

THEOREM 5.8. If $d$ is even, $d \neq 8$, then a set $\mathcal{D}=\left\{A_{1}, \ldots, A_{k}\right\}$ of partitions of $d$ refining $[d / 2, d / 2]$ is the branch data for a connected orientable branched covering of $\mathbf{R} P^{2}$ provided $v(\mathscr{D}) \geqslant 3(d / 2-1)$ and $v(\mathscr{D}) \equiv 0 \bmod 2$.

Proof. Let $d=2 d^{\prime}$. Choose a refinement $A_{i}^{\prime} \cup A_{i}^{\prime \prime}$ of $A_{i}, 1 \leqslant i \leqslant k$. Then for $\mathrm{QQ}^{\prime}=\left\{A_{1}^{\prime}, A_{1}^{\prime \prime}, \ldots, A_{k}^{\prime}, A_{k}^{\prime \prime}\right\}$ one has

$$
\left(\mathscr{D}^{\prime}\right)=\Sigma\left(v\left(A_{i}^{\prime}\right)+v\left(A_{i}^{\prime \prime}\right)\right)=\Sigma v\left(A_{i}\right) \geqslant 3\left(d^{\prime}-1\right) .
$$

By Theorem 5.4, $\mathscr{D}^{\prime}$ is realizable. Composition with the covering $S^{2} \rightarrow \mathbf{R} P^{2}$ as in Proposition 2.7 completes the proof.

Again one has the consequence that, given $d \neq 8$, only finitely many sets 0 of branch data which refine $[d / 2, d / 2]$ and satisfy $v(\mathscr{D}) \equiv 0 \bmod 2$ fail to be realizable by connected orientable branched coverings of $\mathbf{R} P^{2}$.

Complement 5.9. For $d=8$, the collections $\mathscr{D}_{k}=\{[3,1,1,1,1,1],[2,2,2$, $2], \ldots,[2,2,2,2]\}(k \geqslant 2$ partitions $)$ and $\varepsilon_{k}=\{[3,1,2,2],[2,2,2,2], \ldots,[2,2,2,2]\}$ $\left(k \geqslant 2\right.$ partitions) satisfy $v\left(\mathscr{Q}_{k}\right)=v\left(\mathcal{E}_{k}\right)=4 k-2 \geqslant 6=d-2$, yet cannot be the branch data of a connected orientable branched covering of $\mathbf{R} P^{2}$.

Proof. The only refinements of $[4,4]$ for the given data yield the data of Complement 5.6 which are nonrealizable over $S^{2}$.

6. A secondary condition. The purpose of this section is to study the effect of pulling a branched covering $\phi: M \rightarrow S^{2}$ back over itself, leading to a further restriction on the branch data of $\phi$ not implied by the Hurwitz conditions.

Let $\phi: M \rightarrow S^{2}$ be a degree $d$ branched covering with $M$ connected and with branch data $\mathscr{D}=\left\{A_{1}, \ldots, A_{k}\right\}$. Now the restruction $\phi_{0}: M-\phi^{-1} B_{\phi} \rightarrow S^{2}-B_{\phi}$ is an ordinary covering space, and one many form the usual pullback of this covering space over itself

$$
\begin{array}{ccc}
\hat{M}_{0} & \stackrel{\hat{\phi}_{2}}{\rightarrow} & M-\phi^{-1} B_{\phi} \\
\hat{\phi}_{1} \downarrow & & \downarrow \phi_{0} \\
M-\phi^{-1} B_{\phi} & \stackrel{\phi_{0}}{\rightarrow} & S^{2}-B_{\phi}
\end{array}
$$

where $\hat{M}_{0}=\left\{(x, y) \in\left(M-\phi^{-1} B_{\phi}\right) \times\left(M-\phi^{-1} B_{\phi}\right): \phi(x)=\phi(y)\right\}$ and the two maps labeled $\hat{\phi}_{1}$ and $\hat{\phi}_{2}$ are projections on the first and second coordinates, respectively. 
Now the covering $\hat{\phi}_{1}$ may be compactified as described in $\$ 2$ to give a degree $d$ branched covering $\hat{\phi}: \hat{M} \rightarrow M$. Note that $M_{0}$ contains a diagonal copy of $M-\phi^{-1} B_{\phi}$ and hence neither $\hat{M}_{0}$ nor $\hat{M}$ is connected for $d>1$. Also $\hat{\phi}: \hat{M} \rightarrow M$ is not the usual set-theoretic pullback of $\phi: M \rightarrow S^{2}$, which would not be a branched covering in general.

To describe the branch data for $\hat{\phi}$ in terms of the branch data $\mathcal{D}$ of $\phi$, first note that by elementary covering space theory the pullback of $f_{b}: S^{1} \rightarrow S^{1}, f_{b}(z)=z^{b}$ over $f_{a}: S^{1} \rightarrow S^{1}$ consists of $\operatorname{gcd}(a, b)$ circles each mapped by a covering of degree $b / \operatorname{gcd}(a, b)$.

LeMma 6.1. Let $x \in B_{\phi}$ with associated partition $A(x)=\left[a_{1}, \ldots, a_{r}\right]$ and $y \in \phi^{-1}(x)$, with local degree $a_{j}$. Then for the branched covering $\hat{\phi}: \hat{M} \rightarrow M, y$ has the partition

$$
A(y)=[\underbrace{a_{1} / g_{1 j}, \ldots, a_{1} / g_{1 j}}_{g_{i j}}, \ldots, a_{g_{r j}}^{a_{r} / g_{r j}, \ldots, a_{r} / g_{r j}}],
$$

where $g_{i j}=\operatorname{gcd}\left(a_{i}, a_{j}\right)$.

Proof. Simply apply the preceding observation to a small circle going around $y$ in $M$ which maps by a covering of degree $a_{j}$ to a circle going around $x$ in $S^{2}$.

Lemma 6.2. Let $\phi: M \rightarrow S^{2}$ have branch data $\mathscr{D}=\left\{A_{1}, \ldots, A_{k}\right\}$, where $A_{i}=$ $\left[a_{i 1}, \ldots, a_{i r_{i}}\right], 1 \leqslant i \leqslant k$. Then the branched covering $\hat{\phi}: \hat{M} \rightarrow M$ has total branching given by

$$
v(\hat{\mathcal{D}})=\sum_{i=1}^{k} \sum_{m=1}^{r_{i}}\left(d-\sum_{n=1}^{r_{i}} \operatorname{gcd}\left(a_{i m}, a_{i n}\right)\right) \text {. }
$$

Proof. This is an immediate consequence of Lemma 6.1.

If $\mathscr{D}=\left\{A_{1}, \ldots, A_{k}\right\}$ is any collection of partitions of $d, A_{i}\left[a_{\mathrm{i} 1}, \ldots, a_{i r_{\mathrm{i}}}\right], 1 \leqslant i \leqslant k$, it follows that the expression $v(\hat{\mathscr{D}})$ in Lemma 6.2 must satisfy the Hurwitz conditions if $\mathscr{D}$ is the branch data of a branched covering. Now one can rather easily check that $v(\hat{D}) \equiv 0 \bmod 2$. Therefore when $M \neq S^{2}$ no further restriction is imposed. However, if $M=S^{2}$ then $v(\hat{D})$ must also verify an appropriate inequality.

Suppose $M=S^{2}$, so that $\chi(M)=2$. If $\hat{M}$ has $t$ components, then $\chi(\hat{M}) \leqslant 2 t$ and by Proposition 2.4 .

$$
v(\hat{\mathscr{D}}) \geqslant d \chi(M)-2 t=2(d-t) .
$$

The number $t$ of components of $\hat{M}$ is at most the cardinality of $\hat{\phi}^{-1}(y), y \in \phi^{-1} B_{\phi}$, and so

$$
t \leqslant \min _{i, m} \sum_{n=1}^{r_{i}} \operatorname{gcd}\left(a_{i m}, a_{i n}\right) .
$$

Putting together these facts yields the following theorem.

THEOREM 6.3. Let $\mathscr{D}=\left\{A_{1}, \ldots, A_{k}\right\}$ be a collection of partitions of $d$, with $A_{i}=$ $\left[a_{i 1}, \ldots, a_{i r_{i}}\right], 1 \leqslant i \leqslant k$, such that $v(\mathscr{D})=2 d-2$. If $\mathscr{D}$ can be realized by a branched covering of $S^{2}$, then

$$
\sum_{i=1}^{k} \sum_{m=1}^{r_{i}}\left[d-\sum_{n=1}^{r_{i}} \operatorname{gcd}\left(a_{i m}, a_{i n}\right)\right] \geqslant 2\left[d-\min _{i, m} \sum_{n=1}^{r_{i}} \operatorname{gcd}\left(a_{i m}, a_{i n}\right)\right] \text {. }
$$


REMARK. One can check that for any partition $\left[a_{1}, \ldots, a_{r}\right]$ of $d$

$$
\sum_{m=1}^{r}\left[d-\sum_{n=1}^{r} \operatorname{gcd}\left(a_{m}, a_{n}\right)\right] \geqslant d-r\left[\operatorname{gcd}\left(a_{1}, \ldots, a_{r}\right)\right] .
$$

Using this it follows easily that the inequality of Theorem 6.3 always holds when $d$ is prime.

Corollary 6.4. If $d=2 r$, then the data $\mathscr{D}=\{[x, 2 r-x],[2, \ldots, 2],[2, \ldots, 2]\}$, with $x \leqslant r$, is realizable as the branch data for a connected branched covering of $S^{2}$ if and only if $x=r$.

Proof. Interpreting the two sides of the inequality in Theorem 6.3 one finds that the left-hand side is $2 r-(x+g)+2 r-(2 r-x+g)=2 r-2 g$, where $g=$ $\operatorname{gcd}(x, 2 r-x)$. On the other hand the right-hand side is

$$
2[2 r-2(\min (x+g, 2 r-x+g))]=2[2 r-(x+y)]
$$

since $x \leqslant r$. Then Theorem 6.3 states that if $\mathscr{D}$ is realizable, then $2 r-2 g \geqslant 4 r-$ $2(x+g)$. Thus $2 x \geqslant 2 r$. But $x \leqslant r$ implies $x=r$.

It was already shown in $\S 5$ that $\mathscr{D}$ is realizable when $x=r$.

REMARK. In this particular example the pullback $\hat{\phi}: \hat{M} \rightarrow S^{2}$ has exactly two branch points, which must therefore have the same type. One can then conclude $x=r$ simply by determining the corresponding partitions using Lemma 6.1, without actually checking the inequality of Theorem 6.3 .

The inequality of Theorem 6.3 seems to apply to very few examples. In Table 1 are listed all known examples, beyond Corollary 6.4 .

\begin{tabular}{c|c|c} 
& Degree & \multicolumn{1}{|c}{ Data } \\
\hline 1. & 9 & {$[4,4,1],[3,3,3],[2,2,2,2,1]$} \\
\hline 2. & 9 & {$[3,3,3],[3,3,3],[2,2,2,2,1]$} \\
\hline 3. & 10 & {$[4,4,2],[3,3,3,1],[2, \ldots, 2]$} \\
\hline 4. & 16 & {$[5,5,5,1],[3, \ldots, 3,1],[2, \ldots, 2]$} \\
\hline 5. & 16 & {$[4,4,4,4],[3, \ldots, 3,1],[2, \ldots, 2]$} \\
\hline 6. & 18 & {$[4,4,4,4,2],[3, \ldots, 3],[2, \ldots, 2]$} \\
\hline 7. & 21 & {$[5,5,5,5,1],[3, \ldots, 3],[2, \ldots, 2,1]$} \\
\hline 8. & 25 & {$[5, \ldots, 5],[3, \ldots, 3,1],[2, \ldots, 2,1]$} \\
\hline 9. & 36 & {$[5, \ldots, 5,1],[3, \ldots, 3],[2, \ldots, 2]$} \\
\hline 10. & 40 & {$[5, \ldots, 5],[3, \ldots, 3,1],[2, \ldots, 2]$} \\
\hline 11. & 45 & {$[5, \ldots, 5],[3, \ldots, 3],[2, \ldots, 2,1]$} \\
\hline
\end{tabular}

TABLE 1

Theorem 6.3 has a purely algebraic interpretation about doubly transitive subgroups of the symmetric group. Compare $[6,10]$. 
THEOREM 6.5. Suppose $\alpha_{1}, \ldots, \alpha_{k} \in \Sigma_{d}$ such that $\alpha_{1} \cdots \alpha_{k}=1$ and $\left\langle\alpha_{1}, \ldots, \alpha_{r}\right\rangle$ is doubly transitive on $\{1, \ldots, d\}$. Then $\Sigma v\left(\alpha_{i}\right)$ is even and at least $2(d-1)$. If $\sum v\left(\alpha_{i}\right)=2(d-1)$, then

$$
\sum_{i=1}^{k} \sum_{m=1}^{r_{i}}\left(d-\sum_{n=1}^{r_{i}} \operatorname{gcd}\left(a_{i m}, a_{i n}\right)\right) \geqslant 2(d-2)
$$

where $\alpha_{i} \in\left[a_{i 1}, \ldots, a_{i r_{1}}\right], 1 \leqslant i \leqslant k$.

Proof. The existence of $\alpha_{1}, \ldots, \alpha_{k}$ implies the existence of a branched covering $\phi$ : $M \rightarrow S^{2}$, as described in $\S 2$. The first assertion then is just the Hurwitz condition. The hypothesis that $\left\langle\alpha_{1}, \ldots, \alpha_{r}\right\rangle$ is doubly transitive corresponds to the geometric assertion that the pullback $\hat{M}$ has exactly two components. Assuming $\operatorname{\Sigma r}\left(\alpha_{i}\right)=$ $2(d-1)$, one is the diagonal copy of $M=S^{2}$, and the other arises from the transitive action on pairs of elements in $\{1, \ldots, d\}$. Now Theorem 6.3 implies the stated inequality.

Remarks. As examples, for $d=6$ the collections $\{[3,3],[2,2,2],[2,2,2]\}$ and $\{[4,1,1],[3,3],[2,2,2]\}$ both admit transitive realizations, but no such choice of doubly transitive realizations.

There are analogous algebraic results for matrices to be found in [11, Theorem 1, p. 484].

\section{REFERENCES}

1. I. Berstein and A. L. Edmonds, On the classification of generic branched coverings of surfaces, Illinois J. Math. (to appear).

2. G. Boccara, Cycles comme produit de deux permutations de classes données, Discrete Math. 58 (1982), $129-142$.

3. A. L. Edmonds, J. H. Ewing and R. S. Kulkarni, Regular tessellations of surfaces and ( $p, q .2)$-triangle groups, Ann. of Math. (2) 116 (1982), 113-132.

4. Torsion free subgroups of Fuchsian groups and tessellations of surfaces, Invent. Math. 69 (1982), 331-346.

5. C. L. Ezell, Branch point structure of covering maps onto nonorientable surfaces, Trans. Amer. Math. Soc. 243 (1978), 123-133.

6. W. Feit, R. Lyndon and L. L. Scott, A remark about permutations, J. Combin. Theory 18 (1975), 234-235.

7. D. Husemoller, Ramified coverings of Riemann surfaces, Duke Math. J. 29 (1962), 167-174.

8. A. Hurwitz, Über Riemann'sche Flächen mit gegebenen Verzweigungspunkten, Math. Ann. 103 (1891), 1-60.

9. W. S. Massey, Algebraic topologv: An introduction, Harcourt, Brace, and World, New York, 1967.

10. R. Ree, $A$ theorem on permutations, J. Combin. Theory 10 (1971), 174-175.

11. L. L. Scott, Matrices and cohomologv, Ann. of Math. (2) 105 (1977), 473-492.

12. D. Singerman, Subgroups of Fuchsian groups and finite permutation groups, Bull. London Math. Soc. 2 (1970), 319-323.

13. R. E. Stong, Branched coverings, preprint.

14. R. Thom, L'equivalence d'une fonction différentiable et d'un polynome. Topology (2) 3 (1965), 297-307.

Department of Mathematics, Indiana University, Bloomington, Indiana 47405 (Current address of A. L. Edmonds and R. S. Kulkarni)

Department of Mathematics, University of Virginia, Charlottesville, Virginia 22903 (Current address of R. E. Strong) 